\title{
Interoperability and eServices
}

\author{
Aphrodite Tsalgatidou and Eleni Koutrouli \\ Department of Informatics \& Telecommunications, \\ National \& Kapodistrian University of Athens, Greece \\ \{atsalga, ekou\}@di.uoa.gr
}

\begin{abstract}
Services are the building blocks for loosely-coupled, distributed applications based on the Service Oriented Architecture (SOA) principles. One of the major benefits they offer is interoperability both between components of service oriented systems and between different systems. Still, the variety and diversity of implementations and interpretations of SOA and the vast amount of emerging standards hinder interoperability. This paper examines interoperability requirements and related issues in the three major eServices categories: Web, Grid and P2P services. Our aim is to provide the basis for a roadmap towards improving interoperability of eServices.
\end{abstract}

\section{Introduction}

Service Oriented Architectures (SOA) emerged as an evolutionary step from Object and Component based approaches, with the promise to support the loose coupling of system parts and to provide agility, flexibility and cost savings via reusability, interoperability and efficiency. However, the lack of agreement on what constitutes a SOA and the vast amount of emerging standards makes it difficult to understand and utilize the potentials of eServices technologies. In this context, interoperability, which is one of the basic characteristics and benefits of SOA, needs to be further explored in order to find out the open issues and best practices.

In this paper we present the interoperability requirements and related issues in the eService area, considering interoperability both in terms of intra- and inter- paradigm integration. Our goal is to pinpoint the challenges and provide a roadmap of best practices for interoperability. Thus, in section 2 we describe the model of SOA and the three major trends in eServices, i.e. Web, P2P and Grid services with a focus on the standardization efforts. In section 3 we provide the interoperability issues for each eService area and present synergies and integration efforts, whereas in section 4 we give our concluding remarks which are summarized in a table that provides a general interoperability overview for the three areas of eServices.

\section{The eService Model}

According to W3C, a Service Oriented Architecture (SOA) specifies a set of components whose interfaces can be described, published, discovered and invoked over a network. eServices are the building blocks of SOA and are mainly instantiated by Web Services (WS), Grid and P2P Services which are briefly described below. 
Web Services are self-contained, modular applications, accessible via the Web, that provide a set of functionalities to businesses or individuals. We are currently witnessing the rapid development and maturation of a stack of interrelated standards that are defining the WS infrastructure along with a great number of development tools that support the WS development. The key standards for describing, advertising, discovering and binding WS are WSDL, UDDI and SOAP. Besides, there are ongoing standardization efforts in WS composition, orchestration, choreography, security and management (e.g. BPEL4WS, ebXML, WS-Security, etc.). The current Web Services Protocol Stack, along with details regarding the standardization of the various WS protocols can be found in [10].

The term Grid refers to a system that is concerned with the integration, virtualization, and management of services and resources in distributed, heterogeneous environments. The Open Grid Services Architecture (OGSA) [4] is a significant effort by the Global Grid Forum towards the standardization of protocols and interfaces which integrates Grid and WSs. OGSA was initially materialized by the Open Grid Services Infrastructure (OGSI) [14], and more recently by the Web Services Resource Framework (WSRF) proposal [22]. Currently the efforts of the major industry players are targeted to the support of the Globus toolkit [5].

The term "Peer-to-Peer" (P2P) refers to a class of systems and applications that takes advantage of resources - storage, cycles, content, human presence - available at the edges of the Internet. Standards for P2P technologies have not yet been established. Efforts for defining specifications are made by the P2P Working Group, whereas two standardization initiatives are Jabber [8] and JXTA [9].

\section{Interoperability Concerns}

In the eServices domain we view interoperability as the ability of systems, applications and services, to communicate, exchange data and files, work together or operate on behalf of one another. In this section we analyze the specific interoperability issues in the areas of Web, Grid and P2P services (intra-paradigm interoperability) and present synergies between different kinds of eServices (interparadigm interoperability).

Web Services Interoperability. WS promise universal interoperability and integration by establishing commonly agreed protocols for mutually understanding what a service offers and for delivering this functionality in an implementation independent way. Interoperability of legacy applications is also enabled facilitating a seamless integration between heterogeneous systems. Furthermore, new services can be created and dynamically published and discovered without disrupting the existing environment. Thus, WS technology provides a means of interoperating between different software applications, running on a variety of platforms and/or frameworks.

The issue of WS interoperability is addressed at a conceptual level by the W3C's Web Services Architecture (WSA) [23], which identifies the global elements of the global WS network required to ensure interoperability between WS. The various WS standards and enabling technologies address technical level interoperability. The common standards for WS description, publication and invocation (WSDL, UDDI, SOAP) have effectively become de facto standards, and, thus, support basic 
interoperability. However, there is a need for enhanced interoperability in all WS operations. Web Services description should include not only functional characteristics of WSs, but also common semantic information that will enable the meaningful interoperation between different WSs. Semantic description is not supported by current WS description standards (e.g. WSDL, whereas ebXML addresses some semantic issues) although some standardized ontology languages, such as OWL [15] and OWL-S [16], can be used for providing WS description semantics. WS management also requires common management semantics, in relation to management policies and capabilities, to be understood by the requester and provider entities [23]. Furthermore, the wide range of protocols that have been proposed for WS security, reliability and composition hinder interoperability.

Enhancing interoperability between different implementations of WS technologies is the goal of the Web Services Interoperability (WS-I) organization [24], that defines how existing, stable and widely accepted WS standards should be used. Developers should use implementations of standards that have proven interoperability (examples are the WSDL/UDDI/SOAP initiative and integration of SOAP into ebXML), and also keep up with the advancement of standards.

Grid Services Interoperability. Grid services interoperability can be viewed along two different dimensions: between distributed resources in a Grid application, and between different Grid applications.

Interoperability between different distributed resources in a Grid application is a main goal of the various Grid projects despite the different infrastructures they use and the different aspects on which they focus. The OGSA/OGSI and WSRF models provide a framework for Service Oriented Grids aiming at supporting interoperability of distributed services and resources. Grid middleware implementations based on these models provide services and promote interoperability by allowing interoperation of Grid components independently of the operating system and network topology.

Significant effort has also been channelled towards enabling interoperability between different Grid applications. There is a number of approaches that include the definition of a minimal set of Grid services which enable the interoperation of different Grid applications, the integration of different Grid infrastructures, the common Grid resources description [6] and the Semantic Grid [18] which aims at providing interoperability across time as well as space for reuse of services, information, and knowledge.

P2P Services Interoperability. P2P services interoperability, similarly to Grid Services, can be viewed either as: interoperability between different peers in a P2P network, or between different P2P applications.

Interoperability between different peers needs advanced interoperability techniques, since the various heterogeneous nodes of a P2P network need to communicate, exchange content and aggregate their diverse resources, such as computing power or storage space. Most P2P systems use proprietary implementations and protocols for the peers interoperation and functionality. Enhanced interoperability between heterogeneous peers is supported by semantic routing which is recently addressed by researchers, e.g. in Edutella [3].

Interoperability between different P2P applications has not been addressed by early P2P applications, which set up closed peer networks, accessible only to them, 
whereas, currently, only a few P2P systems can interoperate, such as Magi with JXTA. Efforts towards improved interoperability are made by the P2P Working Group. Early attempts on interoperability include Jabber and Groove which are mainly extensible attempts, and not fully interoperable systems. A common infrastructure that will contain the core services of a $\mathrm{P} 2 \mathrm{P}$ application could be a more appropriate approach which is mainly addressed by JXTA and Microsoft's .Net [11].

Inter-paradigm Interoperability. Integration of heterogeneous eServices allows the exploitation of the specific characteristics and benefits of each eServices type by the other eServices, leading to more flexible, efficient and interoperable service-oriented systems. In the following we present synergies between Web, Grid and P2P services and the ways they promote inter-paradigm interoperability in the eServices area.

As already discussed, Grid and WS technologies are in a convergence process, led by the OGSA/OGSI and WSRF proposals. Grid developers can thus exploit the experience of the WS community and concentrate on building the higher-level services that are specific to the Grid application domain. There is a need, however, for WS specifications that could safely be used for building interoperable Web Service Grids, and for this reason a WS specification profile WS-I+ has been proposed in [1]. Another proposal on how Grid applications could be built using existing WS specifications is found in [17].

Recently we are also witnessing a strong movement towards WS and P2P services working in conjunction. Synergies between WS and P2P services include: WS discovery using a P2P-based approach [2], peer discovery in P2P systems using WS as registries, WS interconnection in heterogeneous networks [19], search engines, such as Google, based on P2P and WS, and JXTA projects that incorporate WS.

The techniques that the P2P and Grid models use to handle some of the main issues of distributing computing are discussed in [21] in order to find a common foundation that could alleviate the complexities of each other and fulfill the need for secure, scalable and decentralized collaboration. Another approach to combining aspects of Grid and P2P computing is found in the proposal for a new architecture stack for Grids presented in [13].

The confluence of Web, P2P and Grid services provides the foundation for a common model allowing applications to scale from proximity ad hoc networks to worldwide-scale distributed systems. Some approaches and research projects have started to appear towards supporting this convergence and reusability of the three categories of eServices by providing appropriate models and platforms [12][20].

\section{Concluding Summary}

In this paper we investigated the interoperability potentials and challenges of WS, P2P and Grid services which are the building blocks of SOA and are known as eServices. Our observations are summarized in Table 1 that offers an overview of the interoperability requirements and existing and possible solutions. We believe that the work presented in this paper can be the basis for a roadmap towards improving interoperability in the eServices area which is one of the main benefits of Service Oriented development. 
Table 1. Interoperability approaches and requirements for eServices

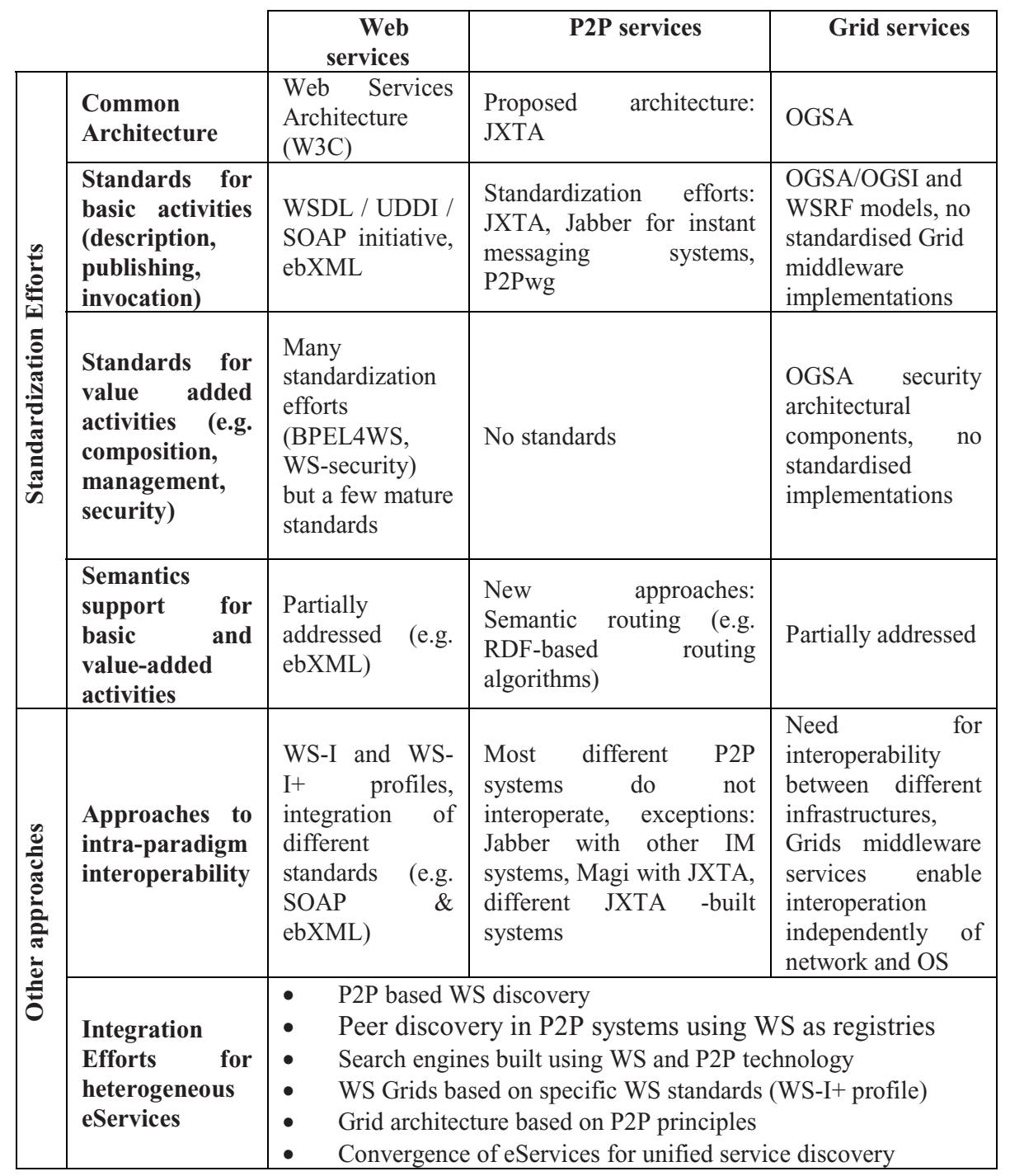

\section{Acknowledgement}

This work has been partially supported by the IST Network of Excellence INTEROP [7]. The authors would like to thank the INTEROP researchers George Athanasopoulos and Vangelis Floros for their contribution in earlier drafts of this paper. 


\section{References}

1. Atkinson, M., DeRoure, D., Dunlop, A., Fox, G., Web Service Grids: An Evolutionary Approach, UK e-Science Technical Report (2004)

2. Banaei-Kashani, F., Ching-Chien Chen, C., Shahabi, C., WSPDS: Web Services Peer-topeer Discovery Service, Proc. of International Symposium on Web Services and Applications (2004)

3. Edutella project, http://edutella.jxta.org

4. Foster, I., Kesselman, C., Nick, J., Tuecke, S.: The Physiology of the Grid: An Open Grid Services Architecture for Distributed Systems Integration, Globus Project (2002)

5. Foster, I., Kesselman, C.: The Globus Toolkit, In Ian Foster and Carl Kesselman, editors, The Grid: Blueprint for a New Computing Infrastructure (1999), 259-278

6. Grid Interoperability Project, http://www.grid-interoperability.org

7. INTEROP Network of Excellence http://interop-noe.org/INTEROP/

8. Jabber (2002), http://www.jabber.org/

9. JXTA, http://www.jxta.org/

10. Lawrence, W., The Web Services Protocol Stack (2005), http://roadmap.cbdiforum.com/ reports/protocols/

11. Microsoft .NET, http://www.microsoft.com/services/net/default.asp

12. Milenkovic, M., Robinson, S., Knauerhase, R., Barkai, D., Garg, S., Tewari, V., Anderson, T., Bowman, M., Intel, Toward Internet Distributed Computing, published at Computer Society (IEEE), (2003), Vol. 36, No. 5, 38-46

13. Next Generation Grid 2nd Group report (2004), http://www.cordis.lu/ist/grids

14. OGSI, http://www-unix.globus.org/toolkit/draft-ggf-ogsi-gridservice-33_2003-06-27.pdf

15. OWL Overview, W3C Recommendation (2004), http://www.w3.org/TR/owl-features/

16. OWL-S 1.0 Release, http://www.daml.org/services/owl-s/1.1/

17. Parastatidis, S., Webber, J., Watson, P., Rischbeck, WS-GAF: A Framework for Building Grid Applications Using Web Services, Journal of Concurrency and Computation: Practice and Experience (2005), 391-417

18. Roure, D., Jennings, N., Shadbolt, N., The Semantic Grid: Past, Present and Future, to appear in IEEE Proceedings March 2005

19. Schattkowsky, T., Loeser, C., Müller, W., Peer-To-Peer Technology for Interconnecting Web Services in Heterogeneous Networks, Proc. of 18th International Conference on Advanced Information Networking and Applications (2004)

20. SODIUM project, http://www.atc.gr/sodium

21. Talia, D., Trunfio, P.: Toward a Synergy Between P2P and Grids, published in IEEE Internet Computing, July 2003, 94-96

22. The WS-Resource Framework (WSRF), http://www.globus.org/wsrf/

23. Web Services Architecture, http://www.w3.org/TR/2004/NOTE-ws-arch-20040211/

24. Web Services Interoperability Organization, http://www.ws-i.org/ 